\title{
COMPOSIÇÃO MINERAL DE FRUTOS DE QUATRO CULTIVARES DE QUIABEIRO
}

\author{
Mineral composition of four cultivars of okra fruits \\ Wagner Ferreira da Mota', Fernando Luiz Finger², Derly José Henriques da Silva², \\ Paulo César Corrêa ${ }^{3}$, Lúcia Pittol Firme ${ }^{4}$, Rosilene Antônio Ribeiro ${ }^{5}$
}

\begin{abstract}
RESUMO
Este experimento foi realizado na Universidade Federal de Viçosa e teve como objetivo avaliar a composição mineral dos frutos de quatro cultivares de quiabo. O delineamento utilizado foi em blocos casualizados, sendo os tratamentos constituídos pelas cultivares Amarelinho, Red Velvet, Star of David e Mammoth Spinless, com cinco blocos. Os maiores teores de cálcio, fósforo, enxofre e magnésio foram encontrado na Cultivar Red Velvet. Os maiores teores de nitrogênio total, amônio e manganês e menor teor de fósforo foram encontrados na cultivar Mammoth Spinless, enquanto nas cultivares Red Velvet e Star of David foram observados os menores teores de nitrogênio total, amônio e manganês. Menores teores de cálcio, enxofre e magnésio foram verificados em conjunto nas cultivares Amarelinho, Star of David e Mammoth Spinless.
\end{abstract}

Termos para indexação: Abelmoschus esculentus (L.), macronutrientes, micronutrientes, Argissolo.

\begin{abstract}
This experiment was carried out at Universidade Federal de Viçosa and had the objective to evaluate the mineral composition of four cultivars of okra fruits. The experimental design was in randomized blocks, where the treatments were composed by the cultivars Amarelinho, Red Velvet, Star of David and Mammoth Spinless, with five blocks. The cultivar Red Velvet had the highest content of calcium, phosphorus, sulfur and magnesium. The cultivar Mammoth Spinless had the highest content of total nitrogen, ammonium and manganese and the lowest content of phosphorus. The cultivars Red Velvet and Star of David had the lowest contents of total nitrogen, ammonium, and manganese. Lower contents of calcium, sulfur and magnesium were present in the cultivars Amarelinho, Star of David and Mammoth Spinless.
\end{abstract}

Index terms: Abelmoschus esculentus (L.), macronutrients, micronutrients, Argisol.

\section{(Recebido em 15 de julho de 2005 e aprovado em 3 de outubro de 2006)}

\section{INTRODUÇÃo}

No Brasil existem condições excelentes para o cultivo do quiabeiro, principalmente no que diz respeito ao clima, sendo popularmente cultivado no nordeste e sudeste. Essa hortaliça possui algumas características desejáveis, tais como: ciclo rápido, custo de produção economicamente viável, resistência à pragas e alto valor alimentício e nutritivo (MOTA et al., 2000).

O valor nutritivo é um atributo de qualidade muito importante, mas é o menos considerado na cadeia de comercialização de hortaliças, uma vez que em muitas delas essa característica não afeta a aparência e a qualidade comestível, ou seja, aroma e/ou textura (CHITARRA \& CHITARRA, 1990). Os componentes responsáveis pela qualidade nutricional dos produtos são vitaminas, minerais, açúcares solúveis, polissacarídeos como amido, fibras, celuloses, hemiceluloses e lignina (CHITARRA \& CHITARRA, 1990; KAYS, 1991).

A nutrição mineral de hortaliças pode influenciar no desenvolvimento vegetal com subseqüentes efeitos na qualidade. $O$ teor de nitrato está relacionado com essa qualidade, pois embora o seu acúmulo, em concentrações excessivas, não afete a produção, ele pode ser um risco para a saúde do consumidor (GRATTAN \& GRIEVE, 1999; RUIZ \& ROMERO, 1999). O acúmulo de nitrato condiciona a conversão em nitrito, a que se ligam as aminas formando nitrosaminas, que são cancerígenas (REZENDE et al., 2001).

O potássio atua como ativador enzimático em mecanismos de síntese e degradação de compostos

\footnotetext{
'Doutor, Professor - Departamento de Ciências Agrárias/DCA - Universidade Estadual de Montes Claros/UNIMONTES - Rua Reinaldo Viana, 2630, Bico da Pedra - 39440.000 - Janaúba, MG - wagner.mota@unimontes.br

${ }^{2}$ Ph. D., Professor Adjunto - Departamento de Fitotecnia/DFT - Universidade Federal de Viçosa/UFV - 36571-000 - Viçosa, MG - ffinger@ufv.br, derly@ufv.br

${ }^{3}$ Doutor, Professor Adjunto - Departamento de Fitotecnia/DFT - Universidade Federal de Viçosa/UFV - 36571-000 - Viçosa, MG -copace@ufv.br

${ }^{4}$ Mestre - Centro de Energia Nuclear na Agricultura/CENA - Universidade de São Paulo/USP - Avenida Centenário, 303 - Cx. P. 96 - $13400-970$ Piracicaba, SP - luciapf@cena.usp.br

${ }^{5}$ Doutora - Departamento de Ciências Agrárias/DCA - Faculdade da Amazônia lesa/FAI - Rua 743, 2043, Cristo Rei - 78995-000 - Vilhena, RO agro@iesavilhe.edu.br
} 
orgânicos, participa no mecanismo de abertura e fechamento dos estômatos e osmorregulação, entre outros processos (MARSCHNER, 1995), sendo, portanto, o cátion mais abundante nos vegetais, afetando o rendimento e a qualidade dos produtos colhidos (DALIPARTHY et al., 1994). O cálcio é o nutriente mais freqüentemente associado com a qualidade dos frutos em geral (FURGUSON, et al., 1999; HOFMAN et al., 2002; SAMS, 1999). O íon cálcio, em concentrações adequadas no tecido, tem sido usado para aumentar a textura e a firmeza, retardar a senescência e reduzir a taxa respiratória e a produção de etileno (HANSON et al., 1993; POOVAIAH, 1986; SAFTNER \& CONWAY, 1998). As pontes de cálcio entre os ácidos pécticos ou entre esses e outros polissacarídeos dificultam o acesso e a ação de enzimas pectolíticas produzidas pelo fruto que causam amaciamento, e daquelas produzidas pelos fungos e bactérias que causam deterioração (CONWAY et al., 1992; HOFMAN et al., 2002. A função dos micronutrientes não está totalmente determinada, no entanto eles têm função vital e produzem efeito estimulante em reações químicas, bioquímicas, sintéticas, metabólicas, fisiológicas e enzimáticas em vegetais e animais (MOHAMED, 2000; SIKORA \& CIESLIK, 1999).

$\mathrm{Na}$ literatura brasileira são escassas as informações quanto aos teores minerais do quiabo no ponto de colheita comercializável, razão pela qual realizou-se o presente trabalho.

\section{MATERIAL E MÉTODOS}

O experimento foi instalado na Horta de Pesquisa da Universidade Federal de Viçosa - UFV, município de Viçosa-MG, no período de janeiro a março de 2001. O solo foi classificado como Argissolo Vermelho-Amarelo Câmbico, fase terraço (EMBRAPA, 1999). A adubação de plantio e em cobertura foi realizada com base no resultado da análise química do solo, mediante interpretação conforme $5^{\text {a }}$ aproximação de adubação para o Estado de Minas Gerais (CFSEMG, 1999). Realizou-se o plantio direto das sementes no campo, recém-preparado (aração e gradagem) e com calagem feita com o resultado da análise do solo, com espaçamento de 1,0 m x 0,30 m, nos genótipos a serem testados. Os frutos de quiabo foram colhidos no ponto de colheita comercial quando estavam tenros. Acondicionaram-se os frutos em caixas plásticas, e em seguida, eles foram transportados para o Laboratório de Pós-Colheita, do Departamento de Fitotecnia, onde foram selecionados.

O experimento foi organizado segundo delineamento experimental em blocos casualizados, sendo os tratamentos constituídos pelas cultivares Amarelinho, Red Velvet, Star of David e Mammoth Spinless, com cinco blocos; a unidade experimental foi composta por 10 frutos.

As determinações de macro e micronutrientes foram efetuadas na matéria seca do tecido vegetal. N-total foi determinado pelo método de Kjeldahl (BREMNER, 1965). As quantificações de P, K, Ca, Mg, S, Fe, Mn, Zn e Cu foram realizadas após a mineralização pela digestão nítricoperclórica. Utilizou-se o método de redução do fosfomolibdato pela vitamina C, para a dosagem colorimétrica de P, conforme Braga \& Defelipo (1974); fotometria de emissão de chama para determinação de K; espectrometria de absorção atômica para as determinações de $\mathrm{Ca}, \mathrm{Mg}$, Fe, $\mathrm{Mn}, \mathrm{Zn}$ e $\mathrm{Cu}$, enquanto o S foi determinado por turbidimetria do sulfato de bario (BLANCHAR et al., 1965).

Os dados foram interpretados por meio de análise de variância. As médias das cultivares foram comparadas, utilizando-se o teste de Tukey ao nível de $5 \%$ de probabilidade.

\section{RESULTADOS E DISCUSSÃO}

O quiabo pode ser classificado com relação à forma da seção transversal em angular e circular (MOTA et al., 2000; MULLER, 1982; PEDROSA et al., 1983). Os frutos das cultivares estudadas são do tipo cápsula, terminados em ponta, e com relação à forma da seção transversal, os frutos das cultivares Star of David, Mammoth Spinless e Red Velvet revelaram elevado a médio nível de angulosidade. A cultivar Amarelinho se caracterizou por possuir seção transversal circular. Ela é a forma preferida pelo mercado brasileiro (MOTA et al., 2000).

Os resultados da composição mineral dos frutos das cultivares de quiabo estudados encontram-se sumarizadas na Tabela 1. O nitrogênio foi o mineral mais abundante nos frutos das cultivares de quiabo, com média geral de 19,4 $\mathrm{g}$ $\mathrm{kg}^{-1}$. O teor médio de nitrogênio total encontrado na cultivar Mammoth Spinless foi 22,7 $\mathrm{g} \mathrm{kg}^{-1}$, sendo, portanto, superior aos observados nas cultivares Red Velvet e Star of David com teores médios de 17,6 $\mathrm{g} \mathrm{kg}^{-1} \mathrm{e} 17,7 \mathrm{~g} \mathrm{~kg}^{-1}$ respectivamente. $\mathrm{Na}$ cultivar Amarelinho, o teor intermediário de nitrogênio foi $19,7 \mathrm{~g} \mathrm{~kg}^{-1}$.

Das frações de nitrogênio que constituem o nitrogênio total, foi observado teor mais elevado para o nitrogênio orgânico amônio com $18,7 \mathrm{~g} \mathrm{~kg}^{-1}$ em relação ao nitrato com $0,6 \mathrm{~g} \mathrm{~kg}^{-1}$. É esperado que, em hortaliças folhosas haja maior acúmulo de nitrato, a exemplo da alface que acumula teores de nitrato da ordem de 1,6 e 1,9 $\mathrm{g} \mathrm{kg}^{-1}$ para as cultivares Elisa e Regina respectivamente 
Tabela 1 - Valores médios dos macronutrientes nitrogênio total, amônio, nitrato, fósforo, potássio, cálcio, magnésio e enxofre; e dos micronutrientes manganês, ferro, zinco e cobre na matéria seca de quatro cultivares de quiabeiro (Viçosa, UFV, 2003).

\begin{tabular}{|c|c|c|c|c|c|c|}
\hline \multirow[b]{2}{*}{ Características } & \multicolumn{6}{|c|}{ Cultivares } \\
\hline & Amarelinho & Red Velvet & $\begin{array}{l}\text { Star of } \\
\text { David }\end{array}$ & $\begin{array}{l}\text { Mammoth } \\
\text { Spinless }\end{array}$ & Média & $\mathrm{CV}(\%)$ \\
\hline \multicolumn{7}{|c|}{ Macronutrientes $-\mathrm{g} \mathrm{kg}^{-1}$} \\
\hline Nitrogênio Total - NT & $19,7 \mathrm{ab}$ & $17,6 \mathrm{~b}$ & $17,7 \mathrm{~b}$ & $22,7 \mathrm{a}$ & 19,4 & 12,70 \\
\hline -Nitrato - NO3 & $0,5 \mathrm{a}$ & $0,7 \mathrm{a}$ & $0,7 \mathrm{a}$ & $0,7 \mathrm{a}$ & 0,6 & 47,91 \\
\hline -Amônio - NH4 & $19,1 \mathrm{ab}$ & $16,9 \mathrm{~b}$ & $17,0 \mathrm{~b}$ & $22,0 \mathrm{a}$ & 18,7 & 13,05 \\
\hline Fósforo - P & $5,1 \mathrm{ab}$ & $5,6 \mathrm{a}$ & $4,8 \mathrm{ab}$ & $4,5 \mathrm{~b}$ & 5,0 & 8,46 \\
\hline Potássio - K & $2,4 \mathrm{a}$ & $2,3 \mathrm{a}$ & $2,6 \mathrm{a}$ & $2,2 \mathrm{a}$ & 2,3 & 5,66 \\
\hline Cálcio - Ca & $5,5 \mathrm{~b}$ & $9,3 \mathrm{a}$ & $5,1 \mathrm{~b}$ & $5,7 \mathrm{~b}$ & 6,4 & 11,50 \\
\hline Magnésio - Mg & $3,3 \mathrm{~b}$ & $5,3 \mathrm{a}$ & $2,9 \mathrm{~b}$ & $3,4 \mathrm{~b}$ & 3,7 & 7,35 \\
\hline Enxofre $-\mathrm{S}$ & $3,6 \mathrm{~b}$ & $4,6 \mathrm{a}$ & $3,3 \mathrm{~b}$ & $3,6 \mathrm{~b}$ & 3,7 & 11,27 \\
\hline \multicolumn{7}{|c|}{ Micronutrientes $-\mathrm{mg} \mathrm{kg}^{-1}$} \\
\hline Manganês - Mn & $51,73 \mathrm{ab}$ & $25,30 \mathrm{~b}$ & $28,82 \mathrm{~b}$ & $76,61 \mathrm{a}$ & 45,61 & 40,16 \\
\hline Ferro - Fé & $35,41 \mathrm{a}$ & $53,50 \mathrm{a}$ & $62,76 \mathrm{a}$ & $30,77 \mathrm{a}$ & 45,61 & 41,80 \\
\hline Zinco - Zn & $34,90 \mathrm{a}$ & $27,34 \mathrm{a}$ & $29,18 \mathrm{a}$ & $40,60 \mathrm{a}$ & 33,00 & 37,29 \\
\hline Cobre $-\mathrm{Cu}$ & $9,43 \mathrm{a}$ & $7,35 \mathrm{a}$ & $10,01 \mathrm{a}$ & $9,67 \mathrm{a}$ & 9,11 & 17,52 \\
\hline
\end{tabular}

As médias seguidas de uma mesma letra, nas linhas, não diferem a $5 \%$ pelo teste de Tukey $(\mathrm{P}>0,05)$.

(CAVARIANNI et al., 2000). Segundo esses autores, isto é uma vantagem qualitativa do quiabeiro, pois $70 \%$ do nitrato ingerido diariamente é originário de hortaliças, caracterizando a importância do consumo de oleráceas com teores reduzidos de nitrato.

Entre as cultivares estudadas, não houve diferença para os teores de nitrato. Para o amônio houve a mesma tendência observada para o nitrogênio total, ou seja, a cultivar Mammoth Spinless, com teor médio de 22,0 $\mathrm{g} \mathrm{kg}^{-1}$, que foi superior aos encontrados nas cultivares Red Velvet e Star of David, com 16,9 e 17,0 $\mathrm{g} \mathrm{kg}^{-1}$ respectivamente, e a cultivar Amarelinho permanecendo em posição intermediária, com 19,1 $\mathrm{g} \mathrm{kg}^{-1}$.

Com exceção do nitrogênio, todas as cultivares examinadas demonstraram maiores teores de fósforo e cálcio do que os outros macronutrientes estudados (Tabela 1), com médias gerais de 5,0 e $6,4 \mathrm{~g} \mathrm{~kg}^{-1}$, respectivamente. Esses valores são superiores aos encontrados em tubérculo de batata, cultivar Superior (WARMAN \& HAVARD, 1999), com teores de fósforo e cálcio de 1,4 e $0,2 \mathrm{~g} \mathrm{~kg}^{-1}$, respectivamente, e em melão cultivar Bônus (CANATO et al., 2001), com 3,2 e 3,3 $\mathrm{g} \mathrm{kg}^{-1}$ respectivamente. Entre as cultivares, foram observados maiores teores de fósforo e cálcio na cultivar Red Velvet com 5,6 e 9,3 $\mathrm{g} \mathrm{kg}^{-1}$, respectivamente. Menor teor de fósforo foi verificado na cultivar Mammoth Spinless com 4,5 $\mathrm{g} \mathrm{kg}^{-1}$, já as cultivares Amarelinho e Star of David se situaram em posição intermediária com relação ao teor de fósforo. Os menores teores de cálcio foram verificados conjuntamente nas cultivares Amarelinho, Star of David e Mammoth Spinless com 5,5; 5,1 e 5,7 $\mathrm{g} \mathrm{kg}^{-1}$, respectivamente. Não houve diferença entre as cultivares com relação ao teor de potássio, ficando em média com teor de $2,3 \mathrm{~g} \mathrm{~kg}^{-1}$.

Os teores relativamente altos do fósforo, especialmente na cultivar Red Velvet, podem estar relacionados ao fato de os frutos de quiabo serem caracterizados como boas fontes de proteína e óleo (MOTA et al., 2000), e estarem em crescimento e desenvolvimento no ponto de colheita, pois esse mineral é essencial na síntese de proteína, síntese e desdobramento de gorduras, participando ainda no processo de divisão celular (MARSCHNER, 1995).

O maior teor de cálcio observado na cultivar Red Velvet, pode ser uma vantagem em relação às demais cultivares com relação à conservação pós-colheita, uma 
vez que o cálcio condiciona incremento na formação de pontes entre os ácidos pécticos e outros ácidos polissacarídeos que atuam como sítios antisenescência. Essas ligações estabilizam a estrutura da parede e da membrana celular, o que dificulta o acesso e, ou interfere na atividade de enzimas responsáveis pelo amaciamento, como a poligalacturonase, e daquelas produzidas por fungos e bactérias que causam deterioração, mantendo os frutos mais firmes e sem desordens fitopatológicas, por mais tempo, principalmente durante a conservação pós-colheita (CONWAY et al., 1992).

Com relação aos macronutrientes potássio, magnésio e enxofre foram observados teores médios gerais de 2,3; 3,7 e 3,7 $\mathrm{g} \mathrm{kg}^{-1}$, respectivamente (Tabela 1). Embora o potássio seja o segundo ou terceiro macronutriente mais absorvido em muitas culturas (MARSCHNER, 1995), verifica-se que o fruto de quiabo não é o dreno preferencial, esse macronutriente foi acumulado em menor proporção. No entanto, o fruto de tomate é um dreno mais forte de potássio, o teor atinge aproximadamente $53,6 \mathrm{~g} \mathrm{~kg}^{-1}$ desse elemento. De acordo com Fernandes (2000), é acumulado em grandes quantidades para uniformizar o amadurecimento e aumentar a acidez do fruto.

Em frutos de quiabo, o ponto de colheita é determinado pela maturidade hortícola, isto é, quando o teor de fibra é menor que $6,5 \%$, estando tenro e com tamanho de 10 a $14 \mathrm{~cm}$ (MULLER, 1982). Nesse estágio, os frutos ainda não atingiram completo crescimento e desenvolvimento, e segundo a literatura eles não são fortes drenos enquanto tenros (COOMBE, 1976; DUZYAMAN, 1997), dessa forma podem acumular mais $\mathrm{K}$ ao continuar o crescimento e desenvolvimento.

O inverso foi observado com relação ao teor de magnésio, onde o teor médio observado por Fernandes (2000), na faixa de $1,8 \mathrm{~g} \mathrm{~kg}^{-1}$, em frutos de tomate, foram inferiores ao observado em frutos de quiabo. Esse resultado é explicado pelo fato de que o magnésio faz parte da estrutura da clorofila, e no ponto de colheita do quiabo os frutos encontram-se verdes, então, com elevado teor de clorofila, ao contrário do tomate onde a coloração, no ponto de colheita, é muito reduzida. A cultivar Red Velvet demonstrou maiores teores de enxofre e magnésio com 4,6 e 5,3 $\mathrm{g} \mathrm{kg}^{-1}$ respectivamente, em relação às demais cultivares que não diferiram entre si, significativamente. $\mathrm{O}$ maior teor de enxofre é coerente com o maior teor de vitamina $\mathrm{C}$ observado na cultivar Red Velvet, pois o enxofre é importante na formação da molécula de ácido ascórbico (LEE \& KADER, 2000).

Os teores dos micronutrientes ferro, zinco e cobre não variaram entre as cultivares estudadas, permanecendo com médias de 45,6, 33,0 e 9,1 mg kg-1 (Tabela 1).
Com relação ao micronutriente manganês, o maior teor foi verificado na cultivar Mammoth Spinless com 76,61 $\mathrm{mg} \mathrm{kg}{ }^{-1}$, enquanto os menores foram identificados nos frutos das cultivares Red Velvet e Star of David com 25,30 e $28,82 \mathrm{mg} \mathrm{kg}^{-1}$, respectivamente. A cultivar Amarelinho situou-se em posição intermediária com teor de $51,73 \mathrm{mg}$ $\mathrm{kg}^{-1}$. Considerando-se que a ação dos micronutrientes ferro, cobre e manganês ocorre na redução dos íons nitrato (SIKORA \& CIESLIK, 1999). Do presente trabalho, depreende-se que o elevado teor de manganês observado na cultivar Mammoth spinless, provavelmente está associado à maior síntese de compostos nitrogenados, haja vista que foi observado, paralelamente, na mesma cultivar maior teor de clorofilas total, a e b, e dos nitrogênios orgânico amônio e total. Provavelmente, devido a um maior teor protéico. Tais teores de manganês são muito superiores aos 0,84 e 5,0 mg kg-1 encontrados por Elless et al. (2000) e Sikora \& Cieslik (1999) em batata e em cenoura, respectivamente. Esse resultado, juntamente com os reduzidos teores de ferro e cobre, evidencia a reduzida síntese de compostos nitrogenados em batata, pois o tubérculo de batata é um órgão quase que exclusivamente de reserva de amido.

\section{CONCLUSÕES}

Foi constatada considerável variabilidade nas características minerais entre as cultivares Amarelinho, Red Velvet, Star of David e Mammoth Spinless.

Algumas características qualitativas importantes na conservação pós-colheita e no consumo "in natura" foram destacadas em algumas cultivares, entre as quais os maiores teores de fósforo e cálcio na cultivar Red Velvet; de nitrogênio, na cultivar Mammoth Spinless.

\section{AGRADECIMENTOS}

À Fundação de Amparo à Pesquisa do Estado de Minas Gerais - FAPEMIG, pela concessão de bolsa de incentivo à pesquisa e ao desenvolvimento tecnológico do Estado de Minas Gerais ao primeiro autor.

\section{REFERÊNCIAS BIBLIOGRÁFICAS}

BLANCHAR, R. W.; REHM, G.; CALDWELL, A. C. Sulfur in plant material by digestion with nitric and perchloric acid. Soil Science Society of America, Madison, v. 29, n. 1, p. 71-72, 1965 .

BRAGA, J. M.; DEFELIPO, B. Determinação espectrofotométrica de fósforo em extratos de solos e plantas. Revista Ceres, Viçosa, v. 21, n. 113, p. 73-85, 1974. 
BREMNER, J. M. Total nitrogen. In: BLACK, C. A. (Ed.) Methods of soil analysis. Madison: American Society of Agronomy, 1965. part. 2, p. 1149-1178.

CANATO, G. H. D.; BARBOSA, J. C.; CECÍLIO FILHO, A. B. Acúmulo de macro e micronutrientes em melão rendilhado cultivado em casa de vegetação. Horticultura Brasileira, Brasília, v. 19, n. 2, jul. 2001. Suplemento. CD-ROM.

CAVARIANNI, R. L.; CAZETTA, J. O.; MAY, A.; BARBOSA, J. C.; CARVALHO FILHO, A. B. Acúmulo e nitrato em cultivares de alface, cultivadas no inverno, em função do ambiente de cultivo. Horticultura Brasileira, Brasília, v. 18, jul. 2000. Suplemento.

CHITARRA, M. I. F.; CHITARRA, A. B. Pós-colheita de frutos e hortaliças: fisiologia e manuseio. Lavras: ESAL/ FAEPE, 1990. 320 p.

COMISSÃO DE FERTILIDADE DO SOLO DO ESTADO DE MINAS GERAIS. Recomendações para o uso de corretivos e fertilizantes em Minas Gerais: $5^{\mathrm{a}}$ aproximação. Viçosa: CFSEMG, 1999. 359 p.

CONWAY, W. S.; SAMS, C. E.; McGUIRE, R. G.; KELMAN, A. Calcium treatment of Apples and Potatoes to reduce postharvest decay. Plant Disease, Saint Paul, v. 76, n. 4, p. 329-334, 1992.

COOMBE, B. G. The development of fleshy fruits. Annual Review Plant Physiology, Palo Alto, v. 27, p. 507-528, 1976.

DALIPARTHY, J.; BARKER, A. V.; MONDAL, S. S. Potassium fractions with other nutrients in crops: a review focusing on the tropics. Journal of Plant Nutrition, Monticello, v. 17, n. 11, p. 1859-1886, 1994.

DUZYAMAN, E. Okra: botany and horticulture. In: JANICK, J. Horticultural reviews. [S.1.: s.n.], 1997. p. 41-72.

ELLESS, M. P.; BLAYLOCK, M. J.; HUANG, J. W.; GUSSMAN, C. D. Plants as a natural source of concentrated mineral nutritional supplements. Food Chemistry, London, v. 71, p. 181-188, 2000.

EMPRESA BRASILEIRA DE PESQUISA AGROPECUÁRIA. Centro Nacional e Pesquisa de Solos. Sistema brasileiro de classificação de solos. Brasília, DF, 1999. $412 \mathrm{p}$.
FERGUSON, I.; VOLZ, R.; WOOLF, A. Preharvest factors affecting physiological disorders of fruit. Postharvest Biology and Technology, Amsterdam, v. 15, p. 255-262, 1999.

FERNANDES, A. F. Fontes de nutrientes influenciando o crescimento, a produtividade e a qualidade de tomate, pepino e alface, cultivados em hidroponia. 2000. $75 \mathrm{f}$. Dissertação (Mestrado em Fitotecnia) - Universidade Federal de Viçosa, Viçosa, 2000.

GRATTAN, S. R.; GRIEVE, C. M. Salinity-mineral nutrient relations in horticultural crops. Scientia Horticulturae, Amsterdam, v. 78, p. 127-157, 1999.

HANSON, E. J.; BEGGS, J. L.; BEAUDRY, R. M. Applying calcium chloride postharvest to improve highbush Blueberry firmness. HortScience, Alexandria, v. 28, n. 10, p. 1033-1034, 1993.

HOFMAN, P. J.; VUTHAPANICH, S.; WHILEY, A. W.; KLIEBER, A.; SIMONS, D. H. Tree yield and fruit minerals concentrations influence "Hass" avocado fruit quality. Scientia Horticulturae, Amsterdam, v. 92, p. 113-123, 2002.

KAYS, S. J. Postharvest physiology of perishable plant products. New York: V. N. Reinhold, 1991. 453 p.

LEE, S. K.; KADER, A. A. Preharvest and postharvest factors influencing vitamin $\mathrm{C}$ content of horticultural crops. Postharvest Biology and Technology, Amsterdam, v. 20, p. 207-220, 2000.

MARSCHNER, H. Mineral nutrition of higher plants. San Diego: Academic, 1995. 889 p.

MOHAMED, A. E. Trace element levels in some kinds of dates. Food Chemistry, London, v. 70, p. 9-12, 2000.

MOTA, W. F. da; FINGER, F. L.; CASALI, V. W. D. Olericultura: melhoramento genético do quiabeiro. Viçosa: UFV, 2000. 144 p.

MULLER, J. J. V. Produção de sementes de quiabo (Abelmoschus esculentus (L) Moench). In: MULLER, J. J. V.; CASALI, V. W. D. (Eds.). Seminários de olericultura. 2. ed. Viçosa: UFV, 1982. v. 1, p. 107-149.

PEDROSA, J. F.; MIZUBUTI, A.; CASALI, V. W. D.; CAMPOS, J. P. Caracterização morfológica de introduções de quiabeiro (Abelmoschus esculentus (L.) MOENCH.). Horticultura Brasileira, Brasília, v. 1, n. 1, p. 14-23, 1983. 
POOVAIAH, B. W. Role of calcium in prolonging storage life of fruits and vegetables. Food Technology, [S.1.], v. 40, p. 86-89, 1986.

REZENDE, A. J.; JUNQUEIRA, A. M. R.; BORGO, L. A. Teores de nitrato observados em couve produzida e comercializada em Brasília, DF. Horticultura Brasileira, Brasília, v. 19, n. 2, jul. 2001. Suplemento. CD-ROM.

RUIZ, J. M.; ROMERO, L. Nitrogen efficiency and metabolism in grafted melon plants. Scientia Horticulturae, Amsterdam, v. 81, p. 113-123, 1999.

SAFTNER, R. A.; CONWAY, W. S. Effects of postharvest calcium and fruit coating treatments on postharvest life, quality maintenance and fruit-surface injury in 'Golden Delicius'
Apples. Journal of the American Society for Horticultural Science, Alexandria, v. 123, n. 2, p. 294-298, 1998.

SAMS, C. E. Preharvest factors affecting postharvest texture. Postharvest Biology and Technology, Amsterdam, v. 15, p. 249-254, 1999.

SIKORA, E.; CIESLIK, E. Correlation between the levels of nitrates and nitrites and the contents of iron, copper and manganese in potato tubers. Food Chemistry, London, v. 67, p. 301-304, 1999.

WARMAN, P. R.; HAVARD, K. A. Yield, vitamin and mineral contents of organically and conventionally grown potatoes and sweet corn. Agriculture, Ecosystems and Environment, [S.1.], v. 68, p. 207-216, 1999. 